\title{
Prevalence of Cardiovascular Disease Risk Factors in the Women Population Covered by Health Centers in Ardabil
}

\author{
Leli Avesta, ${ }^{1}$ Sina Rasoolzadeh, ${ }^{2}$ Mahdi Naeim, ${ }^{3}$ and Aziz Kamran $\mathbb{D}^{2}$ \\ ${ }^{1}$ Department of Cardiology, Faculty of Medicine, Ardabil University of Medical Sciences, Ardabil, Iran \\ ${ }^{2}$ School of Medicine and Allied Medical Sciences, Social Determinants of Health Research Center, \\ Ardabil University of Medical Sciences, Ardabil, Iran \\ ${ }^{3}$ Social Determinants of Health Research Center, Ardabil University of Medical Sciences, Ardabil, Iran \\ Correspondence should be addressed to Aziz Kamran; aziz_kamran@ymail.com
}

Received 16 November 2021; Revised 8 December 2021; Accepted 23 December 2021; Published 27 February 2022

Academic Editor: Thereza Maria Magalhães Moreira

Copyright (C) 2022 Leli Avesta et al. This is an open access article distributed under the Creative Commons Attribution License, which permits unrestricted use, distribution, and reproduction in any medium, provided the original work is properly cited.

\begin{abstract}
Objective. This study aimed to determine the prevalence of cardiovascular risk factors in the population of women aged 30 to 60 years covered by health centers in Ardabil. Methods. This retrospective descriptive-analytical study was conducted on 1006 women aged 30 to 60 years who were covered by Ardabil comprehensive urban health service centers, and they were selected by using the multistage random sampling method. In the first stage, health centers in Ardabil were divided into five geographical areas, and the population covered by each of the five areas was calculated. In the second stage, the number of samples was allocated as a quota in the regions, and in the third stage, in proportion to the population covered by each center in Ardabil, the samples were selected. Women with one of the conditions of pregnancy, lactation, history of kidney disease, known diabetes under medication, history of hepatitis, history of cardiovascular surgery, and history of cancer were excluded from the research process. Results. The average activity of individuals was 24.42 minutes per day. The mean intake of fruits and vegetables was $1.9 \pm 0.9$ and $2.1 \pm 1.07$ unit/day, respectively, and meat was $286.6 \pm 174.6$ grams per week. The mean of HDL, LDL, TG, cholesterol, and FBS were 43.6 $\pm 10.4,101.28 \pm 26.3,159.89 \pm 54.01,185.99 \pm 37.9$, and $94.62 \pm 13.3 \mathrm{mg} / \mathrm{dl}$, respectively. The mean systolic blood pressure (SBP) and diastolic blood pressure (DBP) were 108.14 and $68.26 \mathrm{mmHg}$, respectively. Conclusions. Abdominal obesity (waist above 88), obesity and overweight (high body mass index), high triglycerides, high cholesterol, and LDL and HDL outside the proper range were the most important and risk factors for cardiovascular disease among women.
\end{abstract}

\section{Introduction}

Cardiovascular diseases are one of the most important health problems and one of the most growing diseases. Today, these diseases are associated with a very high prevalence in both developed and developing countries and are considered one of the most important causes of death [1]. According to the World Health Organization, about 30 percent of all deaths are due to cardiovascular disease each year [2]. According to studies conducted in Iran, the highest risk of cardiovascular disease in the population is associated with high blood pressure [3].

The prevalence of hypertension in the adult population over 18 years is between $17.3 \%$ and more than $20 \%$ [4], and about $26.9 \%$ in the population is between 25 and 64 years in Iran [5]. The present study examines the prevalence of cardiovascular risk factors in the female population of Ardabil. Identification of these risk factors can be effective in predicting the likelihood of advanced cardiovascular disease and its complications. Knowing the regional prevalence of these risk factors, appropriate measures to prevent the progression of these diseases, and early treatment can be put on the agenda. There are many risk factors for heart disease: age, sex, smoking, physical inactivity, excessive alcohol consumption, unhealthy diet, obesity, genetic predisposition and family history of cardiovascular disease, hypertension (hypertension), hyperglycemia (diabetes mellitus), high blood cholesterol (hyperlipidemia), undiagnosed celiac disease, psychosocial factors, poor economic status and poverty, low level of education, and air pollution [6]. 
Some of these risk factors, such as age, gender, or family history/genetic predisposition, are immutable. However, many important cardiovascular factors can be corrected by lifestyle changes, social changes, and medication (e.g., prevention of hypertension, hyperlipidemia, and diabetes). People with obesity are at risk for coronary heart disease [7].

Many studies have been conducted over the past years on the prevalence of risk factors for noncommunicable diseases, including cardiovascular disease in Iran and its cities and in other parts of the world. In an honest business study conducted in 2006 in Ardabil province, 29\% of men and 2.6\% of women consumed tobacco products daily and the average body mass index of individuals was reported to be $26.6 \mathrm{~kg} /$ $\mathrm{m}^{2}$. The incidence and increase of obesity have been directly related to high blood pressure in the individuals. Fruit consumption per day was reported to be 1.1 per person, and $33 \%$ had a sedentary lifestyle [8]. Behzad et al. conducted a study in 2015 on 460 heart patients in Babol city who underwent coronary artery bypass graft surgery and found a significant prevalence of blood pressure, diabetes, and obesity and the prevalence of the risk factors was high in women [9]. In a similar study conducted in Tabriz in 2017, the prevalence of general obesity and abdominal obesity was significant. There was a very significant relationship between oxidized LDL (serum oxidized LDL) and aging and also between serum hs-CRP and DBP and triglyceride levels [10]. In the study of Moghimi-Dehkordi et al., overweight and obesity were reported to be $36.9 \%$ and $20.6 \%$ in 2008 in Tehran women, respectively [11].

In general, in similar studies, factors such as smoking, obesity, blood pressure, dietary patterns and mobility, income and economic factors, family history, and laboratory cases such as triglycerides, cholesterol, sodium, vitamin D, and zinc were considered risk factors of cardiovascular disease and were investigated. In the present study, an attempt was made to examine demographic and behavioral variables along with clinical and laboratory cases in a wide range of women in Ardabil.

\section{Method}

This descriptive-analytical study was conducted on 1006 women aged 30 to 60 years who were under the auspices of Ardabil comprehensive urban health service centers, and they were selected by using the multistage sampling method. In the first stage, Ardabil health centers are divided into five geographical areas (Region 1: city center; Region 2: South; Region 3: West; Region 4: North; and Region 5: East) and the population covered by each region was calculated. In the second stage, the number of samples was allocated as a quota in the allocated areas, and in the third stage, by referring to all health centers in Ardabil, in proportion to the population covered by each center, the population was sampled from the share of the allocated area. Finally, 224 persons (22.3\%) from Region 1, 208 (20.7\%) from Region 2, 147 (14.6\%) from Region 3, 205 (20.4\%) from Region 4, and 222 (22.1\%) from Region 5 participated in the present study.

By referring to the databases of comprehensive urban health service centers and using the information systems of the desired databases, demographic information of the referring people during 2019 and 2020 with a history of at least ten years of residence in the area, including age, level of education, marital status, and occupational status; the latest anthropometric data recorded including weight, height, waist, and calculation of body mass index; daily consumption of vegetables and fruits; daily activities of the people; smoking; using other drugs; and the latest test results of the patients, such as fasting blood sugar, cholesterol levels, HDL and LDL, and triglycerides, were collected and evaluated. For all the subjects, three blood pressures recorded in the electronic record during the past year were collected and the mean systole and diastole of these three blood pressures were calculated. According to the latest national protocol for monitoring blood pressure provided by the Office of NonCommunicable Diseases Management of the Ministry of Health and Medical Education, SBP less than 120 and DBP less than $80 \mathrm{mmHg}$ were considered normal blood pressure, SBP between 120 and 139 and DBP between 80 and $90 \mathrm{mmHg}$ were considered prehypertension, and SBP above 140 and DBP above $90 \mathrm{mmHg}$ were considered hypertension. Body mass index (BMI), which describes the status of general obesity, is calculated as weight in kilograms divided by height squared in meters. BMI groups were defined and categorized as low weight (BMI $\left.<18.5 \mathrm{~kg} / \mathrm{m}^{2}\right)$, healthy and normal weight $\left(\mathrm{BMI}=18.5-24.9 \mathrm{~kg} / \mathrm{m}^{2}\right)$, overweight $\left(\mathrm{BMI}=25.0-29.9 \mathrm{~kg} / \mathrm{m}^{2}\right)$, and obesity $\left(\mathrm{BMI}>30 \mathrm{~kg} / \mathrm{m}^{2}\right)$. Abdominal obesity was considered as waist circumference equal to or greater than $88 \mathrm{~cm}$. In clinical trials, the triglyceride level less than $150(\mathrm{TG}<150)$ was considered normal, between 150 and 199 (TG $=150-199)$ borderline, between 200 and 499 (TG =200-499) high, and more than 500 ( TG > 500) was considered too high. Cholesterol less than 200, HDL equal to and above 50, and LDL less than 130 were defined as optimal and normal values $[12,13]$.

Women with a history of pregnancy, lactation, kidney disease, known diabetes under medical treatment, hepatitis, cardiovascular surgery, and cancer and people with incomplete electronic file information were excluded from the study.

To comply with the principles of medical ethics, after obtaining the code of ethics (IR.ARUMS.REC.1399.429) by the Ethics Committee of Ardabil University of Medical Sciences and presenting it to health centers, information of individuals without access to identity information, kept confidential, and the results will be reported without mentioning the name.

Data were collected using SPSS software, version 22, and analyzed using independent statistical tests, one-way analysis of variance, multiple linear regression, and equivalent nonparametric tests with a significance level of less than $5 \%$.

\section{Results}

The majority of participants were married (967, 96.1\%), had high waist size $(590,58.6 \%)$, and were overweight (431, $42.8 \%)$. Table 1 shows the frequency distribution of demographic variables in the study.

Table 2 shows the frequency distribution of clinical laboratory variables of the study. In the present study, the 
TABLE 1: Frequency distribution of the study's demographic variables.

\begin{tabular}{|c|c|c|c|}
\hline \multicolumn{2}{|c|}{ Variable } & \multirow{2}{*}{$\begin{array}{l}N \\
30\end{array}$} & \multirow{2}{*}{$\begin{array}{c}\text { Percent } \\
3.0\end{array}$} \\
\hline Marital status & Single & & \\
\hline Mramtal status & Married & 967 & 96.1 \\
\hline \multirow{2}{*}{ Waist size $(\mathrm{cm})$} & Normal (less than 80) & 416 & 41.4 \\
\hline & High (equal to or above 88 ) & 590 & 58.6 \\
\hline \multirow{7}{*}{ Education } & Illiterate & 66 & 6.6 \\
\hline & Primary & 199 & 19.8 \\
\hline & Middle and high school & 131 & 13 \\
\hline & Diploma & 297 & 29.5 \\
\hline & Bachelor & 274 & 27.2 \\
\hline & Masters & 36 & 3.6 \\
\hline & $\mathrm{PhD}$ & 3 & 0.3 \\
\hline \multirow{3}{*}{ Job } & Housewife & 829 & 82.4 \\
\hline & Governmental employee & 138 & 13.7 \\
\hline & Freelance & 39 & 3.9 \\
\hline \multirow{3}{*}{ Smoking } & Yes & 11 & 1.1 \\
\hline & No & 995 & 98.9 \\
\hline & 1 & 224 & 22.3 \\
\hline \multirow{4}{*}{ Region } & 2 & 208 & $20 / 7$ \\
\hline & 3 & 147 & 14.6 \\
\hline & 4 & 205 & 20.4 \\
\hline & 5 & 222 & 22.1 \\
\hline \multirow{4}{*}{ BMI } & Low weight (less than 18.5) & 1 & $0 / 1$ \\
\hline & Normal (18.5-24.9) & 221 & 22 \\
\hline & Overweight (25-29.9) & 431 & 42.8 \\
\hline & Obese (above 30) & 353 & 35.1 \\
\hline
\end{tabular}

TABLE 2: Frequency distribution of clinical laboratory variables.

\begin{tabular}{|c|c|c|c|}
\hline \multicolumn{2}{|c|}{ Variable } & \multirow{2}{*}{$\begin{array}{c}\text { Number } \\
930\end{array}$} & \multirow{2}{*}{$\begin{array}{c}\% \\
92.4\end{array}$} \\
\hline \multirow{3}{*}{ Systolic blood pressure } & Normal & & \\
\hline & Pre-HTN & 66 & 6.6 \\
\hline & HTN & 10 & 1.0 \\
\hline \multirow{3}{*}{ Diastolic blood pressure } & Normal & 957 & 95.1 \\
\hline & Pre-HTN & 43 & 4.3 \\
\hline & HTN & 6 & 0.6 \\
\hline \multirow{4}{*}{ Triglyceride } & Normal (less than 150) & 439 & 43.6 \\
\hline & Borderline (150-199) & 389 & 38.7 \\
\hline & High (200-499) & 177 & 17.6 \\
\hline & Very high (above 500) & 1 & 0.1 \\
\hline \multirow{2}{*}{ Cholesterol } & Normal (less than 200) & 682 & 67.8 \\
\hline & High (equal to or higher than 200) & 324 & 32.2 \\
\hline \multirow{2}{*}{ HDL } & Low (less than 50) & 777 & 77.2 \\
\hline & Suitable (equal to or higher than 50 ) & 229 & 22.8 \\
\hline \multirow{2}{*}{ LDL } & Normal (less than 130) & 861 & 85.6 \\
\hline & High (equal to or higher than 130 ) & 145 & 14.4 \\
\hline
\end{tabular}

majority of participants had normal SBP (930, 92.4\%), normal DBP $(957,95.1 \%)$, borderline TG (389, 38.7\%), normal Chol $(682,67.8 \%)$, low HDL $(777,77.2 \%)$, and normal LDL (861, 85.6\%).

The mean of age, height, weight, BMI, and waist circumstance were $42.9 \pm 8.4$ years, $161.5 \pm 6.7 \mathrm{~cm}$, $74.2 \pm 11.3 \mathrm{~kg}, \quad 28.5 \pm 5.09 \mathrm{~kg} / \mathrm{m}^{2}$, and $91.3 \pm 10.4 \mathrm{~cm}$, respectively. The mean intake of fruits and vegetables was $1.9 \pm 0.9$ and $2.1 \pm 1.07$ unit/day, respectively, and meat was $286.6 \pm 174.6$ (Table 3 ).

The results showed that there were significant differences in the mean of HDL, LDL, TG, FBS, Chol, SBP, and DBP between the normal and obese participants (Table 4).
The results showed that the linear regression model predicted $41.8 \%$ of changes in SBP (Table 5).

The results showed that the linear regression model predicted $39.6 \%$ of changes in DBP (Table 6).

\section{Discussion and Conclusion}

In terms of body mass index, 431 people (42.8\%) were overweight, which included more frequency. $35.1 \%$ were in the obese group. The average waist size was $91.22 \mathrm{~cm}$, and based on the waist size, $58.6 \%$ had abdominal obesity. According to the results, overweight and general obesity and abdominal obesity are very common in our study 
TABLE 3: The mean and standard deviation of quantitative variables.

\begin{tabular}{|c|c|c|c|c|}
\hline Variable & Mean & SD & The least & The most \\
\hline Age (years) & 42.98 & 8.45 & 30 & 60 \\
\hline Height $(\mathrm{cm})$ & 161.51 & 6.7 & 98 & 190 \\
\hline Weight (kg) & 74.29 & 11.39 & 44 & 126 \\
\hline Waist circumference $(\mathrm{cm})$ & 91.32 & 10.4 & 55 & 125 \\
\hline Physical activity (daily activity rate) (minutes) & 24.42 & 35.82 & 0 & 360 \\
\hline Fruits (units per day) & 1.9 & 0.902 & 0 & 5 \\
\hline Vegetables (units per day) & 2.1 & 1.07 & 0 & 8 \\
\hline Meat (grams per week) & 286.65 & 174.64 & 0 & 1000 \\
\hline $\mathrm{HDL}(\mathrm{mg} / \mathrm{dl})$ & 43.6 & 10.84 & 15 & 95 \\
\hline $\mathrm{LDL}(\mathrm{mg} / \mathrm{dl})$ & 101.28 & 26.39 & 35 & 200 \\
\hline $\mathrm{TG}(\mathrm{mg} / \mathrm{dl})$ & 159.89 & 54.01 & 40 & 523 \\
\hline Cholesterol (mg/dl) & 185.99 & 37.90 & 80 & 323 \\
\hline $\mathrm{FBS}(\mathrm{mg} / \mathrm{dl})$ & 94.62 & 13.38 & 63 & 190 \\
\hline Systolic blood pressure $(\mathrm{mmHg})$ & 108.14 & 10.23 & 85 & 147.67 \\
\hline Diastolic blood pressure (mmHg) & 68.26 & 7.86 & 48.33 & 93.33 \\
\hline BMI $\left(\mathrm{kg} / \mathrm{m}^{2}\right)$ & 28.58 & 5.09 & 17.15 & 46.80 \\
\hline
\end{tabular}

TAвLE 4: The relationship between clinical and laboratory cardiovascular risk factors and BMI.

\begin{tabular}{|c|c|c|c|c|}
\hline Variable & BMI & Mean & SD & $P$ value \\
\hline & Normal & 46.88 & 11.17 & \\
\hline \multirow[t]{3}{*}{ HDL } & Overweight & 43.83 & 10.59 & $<0.001$ \\
\hline & Obese & 41.28 & 10.44 & \\
\hline & Normal & 91.46 & 23.72 & \\
\hline \multirow[t]{3}{*}{ LDL } & Overweight & 100.27 & 25.24 & $<0.001$ \\
\hline & Obese & 108.66 & 27.23 & \\
\hline & Normal & 137.94 & $67 / 44$ & \\
\hline \multirow[t]{3}{*}{ TG } & Overweight & 155.38 & $36 / 47$ & $<0.001$ \\
\hline & Obese & 179.23 & $29 / 60$ & \\
\hline & Normal & 175.68 & 32.47 & \\
\hline \multirow[t]{3}{*}{ Cholesterol } & Overweight & 182.94 & 36.194 & $<0.001$ \\
\hline & Obese & 196.20 & 40.727 & \\
\hline & Normal & 90.91 & 19.692 & \\
\hline \multirow[t]{3}{*}{ FBS } & Overweight & 93.30 & 13.410 & $<0.001$ \\
\hline & Obese & 98.58 & 13.909 & \\
\hline & Normal & 101.93 & 8.432 & \\
\hline \multirow[t]{3}{*}{ Systolic pressure } & Overweight & 106.79 & 8.378 & $<0.001$ \\
\hline & Obese & 113.71 & 10.501 & \\
\hline & Normal & 63.56 & 7.079 & \\
\hline \multirow[t]{2}{*}{ Diastolic pressure } & Overweight & 67.57 & 7.064 & $<0.001$ \\
\hline & Obese & 72.08 & 7.409 & \\
\hline
\end{tabular}

TABLE 5: The multiple linear regression model predicting SBP changes based on variables.

\begin{tabular}{lcccc}
\hline Model & $B$ & Beta & $T$ & $P$ value \\
\hline Constant & 55.58 & & 20.44 & $\leq 0.001$ \\
Age & 0.349 & 0.289 & 10.621 & $\leq 0.001$ \\
Waist & 0.178 & 0.182 & 5.163 & $\leq 0.001$ \\
Fruit & $-1 / 521$ & -0.134 & $-5 / 111$ & $\leq 0.001$ \\
Meat & 0.006 & 0.104 & 4.001 & $\leq 0.001$ \\
LDL & 0.052 & 0.134 & 4.741 & $\leq 0.001$ \\
Cholesterol & 0.016 & 0.061 & 2.203 & $\leq 0.001$ \\
FBS & 0.070 & 0.092 & 3.455 & $\leq 0.001$ \\
BMI & 0.255 & 0.127 & 3.675 & $\leq 0.001$ \\
Cigarettes & 0.693 & 0.117 & 4.781 & Adj. $R^{2}: 0.418$ \\
\hline
\end{tabular}


TABLE 6: The multiple linear regression model predicting DBP changes based on variables.

\begin{tabular}{lcccc}
\hline Model & $B$ & Beta & $T$ & $P$ value \\
\hline Constant & 29.202 & & 13.563 & $\leq 0.001$ \\
Age & 0.227 & 0.244 & 8.84 & $\leq 0.001$ \\
Waist size & 0.820 & 0.108 & 2.825 & $\leq 0.005$ \\
Fruit & -1.000 & -0.115 & -4.271 & $\leq 0.001$ \\
Meat & 0.007 & 0.163 & 6.108 & $\leq 0.001$ \\
LDL & 0.056 & 0.190 & 6.590 & $\leq 0.001$ \\
Cholesterol & 0.013 & 0.61 & 2.149 & $\leq 0.032$ \\
FBS & 0.058 & 0.099 & 3.623 & $\leq 0.001$ \\
Weight & 0.115 & 0.166 & 4.506 & $\leq 0.001$ \\
Exercise & -0.018 & -0.082 & -3.202 & $\leq 0.001$ \\
& \multicolumn{2}{c}{$R^{2}: 0.401$} & Adj. $R^{2}: 0.396$ \\
\hline
\end{tabular}

population. In the study of Savadpour et al., which was performed on 354 people over 30 years old in Ardabil in 2014, the prevalence of overweight and obesity was reported to be $18.1 \%$ and $32.5 \%$, respectively [14]. In the study of Moghimi-Dehkordi et al., overweight and obesity were reported to be $36.9 \%$ and $20.6 \%$ in 2008 in Tehran women, respectively [11]. In the study of Hajian-Tilaki and Heidari on the female population of northern Iran, overweight, obesity, and abdominal obesity were reported to be $34.8 \%$, $18.8 \%$, and $28.3 \%$, respectively [15].

The rate of obesity was higher in our study than these three studies, which indicates a higher rate of obesity and overweight in Ardabil women than women in Tehran and northern Iran, which is necessary to find the cause and plan to remove obstacles in this area. In the study of Heshmat et al. on 5724 adults in five metropolises of Iran, the prevalence of overweight and obesity was reported to be $38.5 \%$ and $19.7 \%$, respectively. Abdominal obesity was reported to be $45.1 \%$ in women and $19.6 \%$ in men based on waist circumference. In this study, the cut-off point for waist size in people with a BMI greater than 30 , for men $99.5 \mathrm{~cm}$ and women $94.25 \mathrm{~cm}$, is presented [16], which is $88 \mathrm{~cm}$ in our study. The prevalence of overweight and general and abdominal obesity is lower in our study.

Our results on obesity rates were even higher than those reported for women in some other countries. In the study by Reynolds et al. of Chinese women, the rates of overweight and obesity were $26.1 \%$ and $5 \%$, respectively [17]. According to the National Health and Nutrition Survey (NHANES), the prevalence of general obesity in US women is $34 \%$ [18]. In a 2006 study by Bahrami et al., the prevalence of overweight and obesity in the Iranian population was $62.2 \%$ and $28 \%$, respectively, and showed that obesity, overweight, and hypertension were as prevalent in Iran as in the United States. But Iranian women are fatter than American women, and it shows that health policymakers need to address this gender gap as a major issue [19].

Regular consumption of fruits and vegetables in the diet has always been one of the important health recommendations [20]. In our study, the average physical activity was 24.42 minutes per day, the average consumption of fruits was 1.9 units per day and vegetables 2.1 units per day, and the amount of red meat consumption was 286.65 grams per week.
In the present study, only $1.1 \%$ were smokers, and among these people, the average smoking was about 15 cigarettes per day. In another report by the World Health Organization, the prevalence of daily smoking was $11.9 \%$ for the total population (20.9\% for men and $2.9 \%$ for women) [21]. In the study of Savadpour et al., which was performed on 354 people over the age of 30 in Ardabil, 19.2\% were smokers [14]. A 1998 study in Turkey found that $49.7 \%$ of men and $11.8 \%$ of women were daily smokers [22]. The rate of smokers in our study is lower than all the studies mentioned. High blood pressure is a major problem due to its association with coronary heart disease, cerebrovascular disease, and kidney disease [23].

In our study, $92.4 \%$ were in the normal range in terms of SBP and $95.1 \%$ in terms of DBP. The mean systolic and diastolic blood pressure of the subjects (including the mean of 3 systolic and diastolic blood pressures recorded in the last year for each person) was 108.14 and $68.26 \mathrm{mmHg}$, respectively. Based on systolic and diastolic blood pressure, respectively, $6.6 \%$ and $4.3 \%$ of the subjects were in the prehypertensive range, and $0.1 \%$ and $0.6 \%$ of the subjects were in the hypertensive group. The highest risk of cardiovascular disease is attributed to high blood pressure in the Iranian population [24].

This is because the prevalence of hypertension in the adult population over 18 years in Iran is between $17.3 \%$ and more than 20\% [4] and about $26.9 \%$ in the population is between 25 and 64 years in Iran. [5] About 6.6 million Iranians between the ages of 25 and 64 had high blood pressure in 2005 [25]. According to a national study, among 69,722 Iranian adults aged 25 to 65 , the prevalence of hypertension was $19.8 \%$ in men and $26.9 \%$ in women [26]. In a study of women over 30 years old in southern Iran, the prevalence of hypertension was reported to be $31.4 \%$ [27], and in the study of Gol et al., the prevalence of hypertensive patients based on SBP and DBP was $11.2 \%$ and $4.6 \%$, respectively [10].

In a national study in Iran, the overall prevalence of systolic, diastolic, and also systolic or diastolic blood pressure was $4.2 \%, 5.4 \%$, and $7.7 \%$, respectively, with no significant difference in sex [28]. Various studies have reported a range of 17 to $50 \%$ control of hypertension in patients with hypertension treated in Iran based on different regions, age, and sex $[3-5,24,25]$. In the present study, the predominant type of HDL dyslipidemia was lower serum. High serum triglycerides, high cholesterol, and high serum LDL were other types of dyslipidemia in the subjects. In Latifi et al.'s study of 1,350 women over the age of 20 in Ahvaz, the prevalence of low HDL, hypertriglyceridemia, cholesterol, and high LDL was 54.7\%, $65.1 \%, 47.5 \%$, and $28.4 \%$, respectively [29]. All listed values except the HDL level are higher than our results. However, in the study of Gol et al., abnormal levels of triglycerides, total cholesterol, LDL, and HDL were reported to be $32.5 \%, 25.7 \%, 17.8 \%$, and $56.6 \%$ in the study population, respectively [10]. The results of our study are less. In the study of Khader et al., it was reported that in Jordanian women aged 25 to 85 years, serum triglyceride, cholesterol, and LDL levels were $38.9 \%, 51 \%$, and $41.4 \%$, 
respectively. However, the rate of people with low serum HDL $(27.9 \%)$ is lower than our study [30].

The results of our study are even higher than those reported for 2601 women over the age of 20 in Turkey, with high triglyceride, cholesterol, and high LDL levels of $12.6 \%$, $13 \%, 11.8 \%$, and low HDL levels of $10.2 \%$, respectively [31]. Comparison of age groups with risk factors showed that smoking, BMI, LDL, TG, cholesterol, FBS, and systolic and diastolic blood pressure should increase with age. Consumption of fruits and vegetables and the level of HDL also decrease with age. In the study of Gol et al., there was a very significant relationship between oxidized LDL (serum oxidized LDL) and increasing age [10]; and the relationship between LDL and age groups in our study is also significant. Obesity is associated with various metabolic disorders and increased cardiovascular mortality [32].

Comparison of BMI groups with risk factors showed that HDL, LDL, TG, FBS, and systolic and diastolic blood pressure should increase with increasing BMI. As mentioned in our study, BMI is significantly associated with aging. Similar to our findings, other studies have shown that obesity is less common in early adulthood, is associated with aging, and progresses more rapidly in women [33-35]. In the study of Mousavinasab et al. on 477 patients over the age of 35 who underwent angiography in Sari in 1994-95, the mean body mass index was 28.38 . Of course, the average BMI in our study was 28.58; considering that in our study only women were included in the study and different studies have shown that in societies the average BMI in women is higher than men, we can say that the comparison of the difference between the means in the two studies is not significant, even though this value is higher in our study. In Sadeghi's commercial study, BMI was significantly associated with an increase in systolic and diastolic blood pressure, which was consistent with a study by the World Health Organization in Asian and African countries [36]. In his study, it was stated that the average body mass index in both sexes increases with age, and at least in the first three decades of life in adults, this increase occurs. But in women, it starts to decrease after the age of 54. Interestingly, in his study, physical activity was negatively associated with abdominal obesity and consumption of fruits and vegetables was not associated with abdominal obesity [8].

In our study, there is no strong relationship between fruit and vegetable consumption and body mass index. In the study of Savadpour et al., there was a strong significant relationship between blood pressure and high BMI and waist size [14]. Comparison of region and location with risk factors showed that consumption of fruits, vegetables, and red meat, HDL, LDL, TG, cholesterol, and systolic and diastolic blood pressure have a significant relationship with the location of people in Ardabil.

Comparison of education levels with risk factors showed that with increasing education, smoking and LDL, TG, cholesterol, FBS, and systolic and diastolic blood pressure decreased and HDL and consumption of red meat, vegetables, and fruits increased, indicating the effect of education on the level of knowledge and awareness of people and also the amount of their income. In our study, smoking was higher in single people than married people, and on the other hand, LDL, triglyceride, cholesterol, FBS, and systolic and diastolic blood pressure were higher in married people than in single people. Consumption of fruits, meat, and vegetables, HDL, and systolic and diastolic blood pressure were significantly higher among employed people than unemployed and housewives. In a review of the cardiovascular disease outlook over the past 40 years in Iran, the prevalence of cardiovascular risk factors including hypertension, diabetes mellitus, high LDL, low HDL, hypertriglyceridemia, hypercholesterolemia, obesity, and smoking among the population is currently $42.2 \%, 18.7 \%, 58.9 \%$, $52.3 \%, 52.7 \%, 65.4 \%, 26.4 \%$, and $13 \%$, respectively [16]. The risk of cardiovascular disease associated with diabetes, hypertension, smoking history, abdominal obesity, and high LDL in Iranians was 9.9\%, 36\%, 5.5\%, 18.9\% and 24.1\% [37]. Considering the prevalence of cardiovascular diseases and their risk factors, it has been concluded that the prevention and control of these modifiable risk factors can prevent up to $80 \%$ of cardiovascular diseases [14].

The models studied in our study show that the variables studied in our study predict $41.8 \%$ of changes in SBP and $39.6 \%$ of changes in DBP. In the study of Mousavinasab et al., in univariate logistic regression analysis, the variables of age, sex, smoking, fasting blood sugar, HDL, and triglyceride were significantly associated with increased vasoconstriction above $50 \%$. This means that for every ten years of age, the risk of coronary artery disease is $58 \%$, and men are 4.91 times more likely than women to have coronary artery disease. Smoking also increases the chance of coronary artery disease by more than $50 \%$. HDL cholesterol below 40 for men and below 50 for women, fasting blood sugar above 110, and triglycerides above 150 also increase the chance of coronary artery disease by over $50 \%, 75 \%$, and $61 \%$, respectively.

In the multivariate logistic regression model, the odds ratio of sex, HDL, fasting blood sugar, triglyceride, and age increased and the smoking variable lost its significance [38]. Based on these results, it seems that facility-based opportunistic screening for cardiovascular risk factors is a viable option, and according to similar results in other studies, it is suggested that this is a regular practice in all PHCs in Ardabil. [15] Our findings provide evidence for health policymakers and other health officials about lifestyle problems in the study population. If an effective prevention strategy is not implemented, more disease burden is expected. Although short-term training programs have been shown to be effective in improving lifestyles, a sustainable education strategy and cost-saving policies supported by continuing media education and school-based education programs could be the starting point for a possible national program to control noncommunicable cardiovascular diseases $[20,39,40]$.

4.1. Limitations. Because this study is retrospective, detailed information on how and what type of physical activity people do and the number of days of the week that people have effective and regular physical activity is not available. 
Another limitation of this study is the limitation of recording people's tests in electronic records (for example, items such as the levels of vitamins D, E, calcium, sodium, potassium, and alkaline phosphatase, which are important and effective factors associated with the disease. Are cardiovascular, not routinely measured and recorded in the population), and it is suggested that future research would examine the relationship between these and cardiovascular hazards.

4.2. Conclusion. According to the results obtained during the past years, the amount of smoking in the female population of Ardabil has been decreasing and the daily consumption of fruits in the diet of people has increased.

However, the rate of abdominal obesity (waist circumference above 88), obesity and overweight (high body mass index), high triglycerides, high cholesterol, and HDL and LDL outside the proper range are the most important and risk factors for cardiovascular disease in the women population of Ardabil.

\section{Abbreviations}

HTN: Hypertension

BMI: Body mass index

CVD: Cardiovascular disease

HDL: High-density lipoprotein

LDL: Low-density lipoprotein

TG: Triglycerides.

\section{Data Availability}

The original data are available on request to the corresponding author, after the manuscript published. Whatever, we also considered to provide the original data in public repositories.

\section{Ethical Approval}

This study was examined by the Ethics Committee of the Ardabil University of Medical Sciences and received approval with the ethics code of IR.ARUMS.REC.1399.429. Participating to the study was voluntarily, and it was based solely on the participants' full consent. Details of the sampling method and data gathering have been approved by the university's ethical committee.

\section{Consent}

Not applicable.

\section{Conflicts of Interest}

None of the authors have any conflicts of interest to declare.

\section{Authors' Contributions}

$\mathrm{AK}$ is the correspondence author of this study, responsible for designing, organizing, and planning this study. AK, LV, $\mathrm{SR}$, and $\mathrm{MN}$ provided feedback and reviewed the manuscript to aid in revisions. $\mathrm{AK}, \mathrm{LA}$, and $\mathrm{MN}$ were responsible for data collection and analyzing, designing, and planning this study. All the authors have read and approved the manuscript.

\section{References}

[1] E. J. Benjamin, S. S. Virani, C. W. Callaway et al., "Heart disease and stroke statistics-2018 update: a report from the American Heart Association," Circulation, vol. 137, no. 12, pp. e67-492, 2018.

[2] World Health Organization, Global Tuberculosis Control: WHO Report 2010, World Health Organization, Geneva, Switzerland, 2010.

[3] N. Sarrafzadegan and N. Mohammmadifard, "Cardiovascular disease in Iran in the last 40 years: prevalence, mortality, morbidity, challenges and strategies for cardiovascular prevention," Archives of Iranian Medicine, vol. 22, no. 4, pp. 204-210, 2019.

[4] S. Shirani, R. Kelishadi, N. Sarrafzadegan et al., "Awareness, treatment and control of hypertension, dyslipidaemia and diabetes mellitus in an Iranian population: the IHHP study," Eastern Mediterranean Health Journal, vol. 15, no. 6, pp. 1455-1463, 2009.

[5] A. Gandomkar, H. Poustchi, F. Malekzadeh et al., "Prevalence, awareness, treatment, control, and correlates of hypertension in the pars cohort study," Archives of Iranian Medicine, vol. 21, no. 8, pp. 335-343, 2018.

[6] R. Micha, G. Michas, and D. Mozaffarian, "Unprocessed red and processed meats and risk of coronary artery disease and type 2 diabetes-an updated review of the evidence," Current Atherosclerosis Reports, vol. 14, no. 6, pp. 515-524, 2012.

[7] R. H. Eckel and November, "Obesity and heart disease," Circulation, vol. 96, no. 9, pp. 3248-3250, 1997.

[8] H. Sadeghi-Bazargani, H. Jafarzadeh, M. Fallah et al., "Risk factor investigation for cardiovascular health through WHO STEPS approach in Ardabil, Iran," Vascular Health and Risk Management, vol. 7, 2011.

[9] C. Behzad, S. Zakeri, and H. Vafaey, "An evaluation of the risk factors of coronary artery disease in patients undergoing coronary artery bypass graft surgery in Babol," Journal of Babol University of Medical Sciences, vol. 21, pp. 6-10, 2019.

[10] R. M. Gol, M. Rafraf, and M. Asghari Jafarabadi, "Evaluation of cardiovascular risk factors in women referring to health centers in Tabriz, Iran, 2017," Health Promotion Perspectives, vol. 8, no. 4, pp. 315-322, 2018.

[11] A. Safaee, A. Pourhoseingholi, M. Pourhoseingholi et al., "Overweight and obesity and related factors in urban Iranian population aged between 20 to 84 years," Annals of Medical and Health Sciences Research, vol. 3, no. 2, pp. 171-176, 2013.

[12] L. K. Mahan and J. L. Raymond, Krause's Food \& The Nutrition Care Process, Elsevier, St. Louis, MO, USA, 2016.

[13] W. T. Friedewald, R. I. Levy, and D. S. Fredrickson, "Estimation of the concentration of low-density lipoprotein cholesterol in plasma, without use of the preparative ultracentrifuge," Clinical Chemistry, vol. 18, no. 6, pp. 499-502, 1972.

[14] M. T. Savadpour, S. Gh, M. Mohebi, A. Kamran, and A. Dargahi, "Prevalence of hypertension and cardiovascular risk factors among adults in urban populations-Iran," Archives of Hygiene Sciences, vol. 3, no. 2, pp. 44-49, 2014.

[15] K. O. Hajian-Tilaki and B. Heidari, "Prevalence of obesity, central obesity and the associated factors in urban population aged 20-70 years, in the north of Iran: a population-based study and regression approach," Obesity Reviews, vol. 8, no. 1, pp. 3-10, 2007. 
[16] R. Heshmat, P. Khashayar, H. R. Meybodi, M. R. Homami, and B. Larijani, "The appropriate waist circumference cut-off for Iranian population," Acta Medica Indonesiana, vol. 42, no. 4, pp. 209-215, 2010.

[17] K. Reynolds, D. Gu, P. K. Whelton et al., "Prevalence and risk factors of overweight and obesity in China," Obesity, vol. 15, no. 1, pp. 10-18, 2007.

[18] C. L. Ogden, M. D. Carroll, L. R. Curtin, M. A. McDowell, C. J. Tabak, and K. M. Flegal, "Prevalence of overweight and obesity in the United States, 1999-2004," JAMA, vol. 295, no. 13, pp. 1549-1555, 2006.

[19] H. Bahrami, M. Sadatsafavi, A. Pourshams et al., "Obesity and hypertension in an Iranian cohort study; Iranian women experience higher rates of obesity and hypertension than American women," BMC Public Health, vol. 6, no. 1, p. 158, 2006.

[20] A. H. Lichtenstein, L. J. Appel, M. Brands et al., "Diet and lifestyle recommendations revision 2006," Circulation, vol. 114, no. 1, pp. 82-96, 2006.

[21] WHO Report on the Global Tobacco Epidemic, The MPOWER Package, World Health Organization, Geneva, Switzerland, 2008.

[22] A. I. Bozkurt, S. Şahinöz, B. Özçırpıcı et al., "Patterns of active and passive smoking, and associated factors, in the south-east anatolian project (SEAP) region in Turkey," BMC Public Health, vol. 6, no. 1, p. 15, 2006.

[23] S. Sinha, K. Kar, M. Soren, and A. Dasgupta, "hsCRP in prehypertension and hypertension: a prospective study in southern Asian region," International Journal of Research in Medical Sciences, vol. 2, no. 4, pp. 1402-1407, 2017.

[24] N. Sarrafzadegan, M. Talaei, M. Sadeghi et al., "The Isfahan cohort study: rationale, methods and main findings," Journal of Human Hypertension, vol. 25, no. 9, pp. 545-553, 2011.

[25] A. Esteghamati, M. Abbasi, S. Alikhani et al., "Prevalence, awareness, treatment, and risk factors associated with hypertension in the Iranian population: the national survey of risk factors for noncommunicable diseases of Iran," American Journal of Hypertension, vol. 21, no. 6, pp. 620-626, 2008.

[26] M. Janghorbani, M. Amini, M. M. Gouya, A. Delavari, S. Alikhani, and A. Mahdavi, "Nationwide survey of prevalence and risk factors of prehypertension and hypertension in Iranian adults," Journal of Hypertension, vol. 26, no. 3, pp. 419-426, 2008.

[27] K. Rahmanian and M. Shojaie, "The prevalence of pre-hypertension and its association to established cardiovascular risk factors in south of Iran," BMC Research Notes, vol. 5, no. 1, p. 386, 2012.

[28] R. Kelishadi, G. Ardalan, R. Gheiratmand et al., "Blood pressure and its influencing factors in a national representative sample of Iranian children and adolescents: the CASPIAN study," European Journal of Cardiovascular Prevention \& Rehabilitation, vol. 13, no. 6, pp. 956-963, 2006.

[29] S. M. Latifi, L. Moradi, H. Shahbazian, and A. M. Aleali, "A study of the prevalence of dyslipidemia among the adult population of Ahvaz, Iran," Diabetes \& Metabolic Syndrome: Clinical Research Reviews, vol. 10, no. 4, pp. 190-193, 2016.

[30] Y. S. Khader, A. Batieha, M. El-Khateeb, M. Al Omari, and K. Ajlouni, "Prevalence of dyslipidemia and its associated factors among Jordanian adults," Journal of Clinical Lipidology, vol. 4, no. 1, pp. 53-58, 2010.

[31] C. Erem, A. Hacihasanoglu, O. Deger, M. Kocak, and M. Topbas, "Prevalence of dyslipidemia and associated risk factors among Turkish adults: trabzon lipid study," Endocrine, vol. 34, no. 1-3, pp. 36-51, 2008.
[32] A. Khoshdel, S. M. Seyed Jafari, S. T. Heydari, F. Abtahi, A. Abdi Ardekani, and F. Jabbary Lak, "The prevalence of cardiovascular disease risk factors, and metabolic syndrome amongbiranian military parachutists," International Cardiovascular Research Journal, vol. 6, no. 2, pp. 51-55, 2012.

[33] M. Seyffarshad, A. Kousha, M. Pourdowlati, M. Karamouz, M. Farahbakhsh, and S. Hakimi, "Cardiac risk factor analysis in east Azerbaijan, Iran," The Cardiology, vol. 3, no. 1, pp. 1-4, 2007.

[34] Iranian MOH, National NCD Health Survey 2005, Iranian Ministry of Health, Tehran, Iran, 2002.

[35] S. Dastgiri, R. Mahdavi, H. TuTunchi, and E. Faramarzi, "Prevalence of obesity, food choices and socio-economic status: a cross-sectional study in the north-west of Iran," Public Health Nutrition, vol. 9, no. 8, pp. 996-1000, 2006.

[36] F. Tesfaye, N. G. Nawi, H. Van Minh et al., "Association between body mass index and blood pressure across three populations in Africa and Asia," Journal of Human Hypertension, vol. 21, no. 1, pp. 28-37, 2007.

[37] M. Sadeghi, M. Talaei, S. Oveisgharan et al., "The cumulative incidence of conventional risk factors of cardiovascular disease and their population attributable risk in an Iranian population: the Isfahan cohort study," Advanced Biomedical Research, vol. 3, pp. 242-245, 2014.

[38] N. Mousavinasab, J. Y. Cherat, B. Bagheri, F.-S. Bakhti, and Z. Bakhti, "Identifying the risk factors for cardiovascular disease in individuals aged above 35 years using logistic regression model," Journal of Mazandaran University of Medical Sciences, vol. 26, pp. 50-56, 2017.

[39] R. Pazoki, I. Nabipour, N. Seyednezami, and S. R. Imami, "Effects of a community-based healthy heart program on increasing healthy women's physical activity: a randomized controlled trial guided by community-based participatory research (CBPR)," BMC Public Health, vol. 7, no. 1, p. 216, 2007.

[40] D. S. Sartorelli, E. C. Sciarra, L. J. Franco, and M. A. Cardoso, "Beneficial effects of short-term nutritional counselling at the primary health-care level among Brazilian adults," Public Health Nutrition, vol. 8, no. 7, pp. 820-825, 2005. 